ARTICLE

\title{
Quantum-dot and organic hybrid tandem light-emitting diodes with multi-functionality of full-color-tunability and white-light-emission
}

Heng Zhang ${ }^{1}$, Qiang Su$^{1} \&$ Shuming Chen (1) ${ }^{1 凶}$

Realizing of full-color quantum-dot LED display remains a challenge because of the poor stability of the blue quantum-dot and the immature inkjet-printing color patterning technology. Here, we develop a multifunctional tandem LED by stacking a yellow quantum-dot LED with a blue organic LED using an indium-zinc oxide intermediate connecting electrode. Under parallel connection and alternate-current driving, the tandem LED is full-color-tunable, which can emit red, green and blue primary colors as well as arbitrary colors that cover a 63\% National Television System Committee color triangle. Under series connection and direct current driving, the tandem LED can emit efficient white light with a high brightness of $107000 \mathrm{~cd} \mathrm{~m}^{-2}$ and a maximum external quantum efficiency up to $26.02 \%$. The demonstrated hybrid tandem LED, with multi-functionality of full-color-tunability and white lightemission, could find potential applications in both full-color-display and solid-state-lighting.

\footnotetext{
${ }^{1}$ Department of Electrical and Electronic Engineering, Southern University of Science and Technology, 518055 Shenzhen, P. R. China. ${ }^{\bowtie}$ email: chen.sm@sustech.edu.cn
} 
C dSe-based quantum-dot light-emitting diodes (QLEDs) have been extensively explored for applications in display and lighting due to their unique merits of high color saturation, tunable emission color, high brightness, and simple solution processability ${ }^{1-12}$. Recent advances have enabled QLEDs to exhibit high external quantum efficiency (EQE) and long operational lifetime. For instance, the EQEs of the state-of-the-art red- (R-), green- (G-), and blue- (B-) QLEDs are higher than $20.5 \%^{1,3,13,14}, 22 \%^{3,15-18}$, and $18 \%^{14,19}$, respectively, while the $T_{50}$ lifetime of R- and G-QLEDs are longer than 26,500 (refs. ${ }^{3,20}$ ) and $25000 \mathrm{~h}$ (refs. ${ }^{3,18}$ ) at an initial brightness of $1000 \mathrm{~cd} \mathrm{~m}^{-2}$. Although the efficiency of QLEDs could meet the requirements of display applications, the realization of QLED full-color display remains challenging.

One reason is that the B-QLEDs are unstable, with a short $T_{50}$ lifetime of $\sim 200 \mathrm{~h}$ at an initial brightness of $1000 \mathrm{~cd} \mathrm{~m}^{-2}$ (refs. ${ }^{3,21}$ ), which lags far behind those of R- and G-QLEDs. While the stability of B-QLEDs is being questioned, its organic cousins-the fluorescent organic light-emitting diodes (FLOLEDs), are relatively stable and have been applied in displays for years. By substituting the B-QLEDs with B-OLEDs, a hybrid display could be realized, which can enjoy both the high saturation of QLEDs as well as the high stability of OLEDs. However, promoting the marriage between QLEDs and OLEDs could be difficult, since they come from different families.

Another reason is that the inkjet printing, which is used to deposit and pattern the light-emitting layers (EMLs), has many shortcomings such as low resolution, poor uniformity, and is far from mature for mass-producing QLED displays ${ }^{22-25}$. Instead of patterning the EMLs directly, the R/G/B side-by-side color pixels can also be realized by combining the white devices with the patterned color filters $(\mathrm{CF})^{26,27}$. The "white+CF" scheme has been proven to be an effective strategy for manufacturing largearea OLED televisions and high-resolution OLED microdisplays ${ }^{26-29}$. However, the introduction of CFs significantly reduces the brightness for more than $2 / 3$ of the white light is absorbed by the CFs. To eliminate the absorbtive CFs, it is desirable to develop a color-tunable device that can emit R, G, and B primary colors through the variation of driving conditions. By emitting the $R, G$, and $\mathrm{B}$ colors time-sequentially, a full-color image can be displayed. In this case, neither CFs nor EMLs patterning are needed. Moreover, the pixel density and fill factor of the display can be increased by threefold, since a single color-tunable pixel can take the job of three side-by-side R/G/B pixels, as shown in Supplementary Fig. 1. Driven by these benefits, color-tunable devices have long been pursued. In 1990s, Forrest and co-authors first obtained full-color-tunable OLEDs by vertically stacking R-, G-, and $\mathrm{B}-\mathrm{OLEDs} \mathrm{s}^{30,31}$, but it requires four independently addressable electrodes to drive these devices, which complicates the fabrication and the drive circuits. To make it practical for display application, one has to invent a new architecture with only two electrodes. Unfortunately, all reported two-terminal devices can only emit two primary colors at most ${ }^{32-36}$, making them useless for full-color displays.

To address aforementioned challenges, we hereby develop a two-terminal and full-color-tunable device, which is realized by stacking a yellow (Y)-QLED with a B-OLED using an indium-zinc oxide (IZO) intermediate connecting electrode (ICE). The fluorescent organic molecules are adopted as the Bemitters to substitute the unstable B-QDs. With this novel tandem structure, QLED and OLED can be well integrated, either in parallel or in series, in a single two-terminal architecture. By varying the driving alternate-current $(\mathrm{AC})$ signals, the device can emit R, G, and B primary colors as well as arbitrary colors, making it an ideal candidate for CF-free and EML-patterning-free fullcolor displays. Besides full-color-tunability, the device can also emit efficient white light with a high brightness of $107000 \mathrm{~cd} \mathrm{~m}^{-2}$ and an EQE up to $26.02 \%$ when under direct-current (DC) driving. With a novel AC driving, the white LED can emit stable colors with 1931 Commission International de I'Eclairage (CIE) coordinates fixed at $(0.34,0.36)$ over a wide range of brightness $\left(1000-50,000 \mathrm{~cd} \mathrm{~m}^{-2}\right)$. Also, the color coordinates can be tuned to trace the blackbody locus over a wide range of correlated color temperature $(1500-10,000 \mathrm{~K})$. We believe that our demonstrated hybrid tandem LED, with multi-functionality of full-color tunability and white light emission, could find potential applications in both full-color display and solid-state lighting.

\section{Results}

Device structure and functionalities. As shown in Fig. 1, the multifunctional tandem LED consists of a bottom CdSe-based YQLED and a top MADN:DSA-Ph-based FL B-OLED, which are vertically stacked and connected through an ICE. To obtain higher efficiency, the FL B-OLED can be replaced by a mCPPO1:FIrPicbased phosphorescent $(\mathrm{Ph}) \mathrm{B}$-OLED. Compared with conventional tandem devices that usually employ $\mathrm{p} / \mathrm{n}$ hetero-layers as the connection media14,17,37,38, our device adopts a conductive and transparent IZO as the ICE, which can enable an effective electrical connection as well as an efficient optical coupling. Also, the IZO could promote the hybrid marriage between QLED and OLED. As shown in Fig. 1b, with IZO as the ICE, charge carriers can be efficiently injected into both Y-QLED and B-OLED, leading to the efficient light emission. Moreover, the IZO can be extracted as an independent electrode, thereby allowing us to control the devices in different ways, as will be discussed later.

The IZO was deposited by sputtering. To protect the quantumdot EML from ion bombardment damage during sputtering process, an ultra-thin $(2 \mathrm{~nm}) \mathrm{Al}$ was introduced, which is previously demonstrated as an effective buffer layer for transparent $\mathrm{QLED}^{39}$. Coupled with the protection of $\mathrm{ZnMgO}$ electron transport layer, the bombardment damage could be minimized ${ }^{40}$. Indeed, as shown in Supplementary Fig. 2, with the sputtered $80-\mathrm{nm}$ IZO as the top electrode, the transparent RQLED exhibits an EQE of $11.4 \%$, which is slightly smaller than $12.5 \%$ of the conventional device, implying the good feasibility of using IZO as the top electrode for damage-free QLED.

The adoption of IZO as intermediate electrode, which enables the devices to be connected either in series or in parallel, is a key strategy for the realization of multifunctional tandem LED. Conventionally, with bottom ITO as an anode and top $\mathrm{Al}$ as a cathode, the B-OLED and Y-QLED are connected in series, and under the driving of a DC source, efficient white light emission can be obtained. Also, the devices can be connected in parallel by combing the bottom ITO and the top $\mathrm{Al}$ as one electrode, and extracting the IZO as the counter electrode, as shown in Fig. 1a. In this way, the Y-QLED is reversely biased while the B-OLED is forwardly driven, and thus, both devices can be independently addressed by the polarity of the driving signals, consequently enabling a color-tunable device, as will be discussed in the next section.

Full-color-tunable LED. Under parallel connection and AC driving, the Y-QLED is turned-on by the negative pulses while the B-OLED is lit-up by the positive ones, and thus the tandem LED alternately emits the $\mathrm{Y}$ and $\mathrm{B}$ colors. To display the full-color, a third color should be introduced. To this end, the Y-QLED is specially configured, with its EML consisting of mixing R- and G-QDs. As shown in Fig. 2a, at a small driving voltage, most charge carriers tend to inject and recombine on the R-QDs due to their small injection barrier. The exciton recombination zone could gradually migrate from the R-QDs to the G-QDs when the driving voltage is increased. Therefore, the emitting color of the Y-QLED 


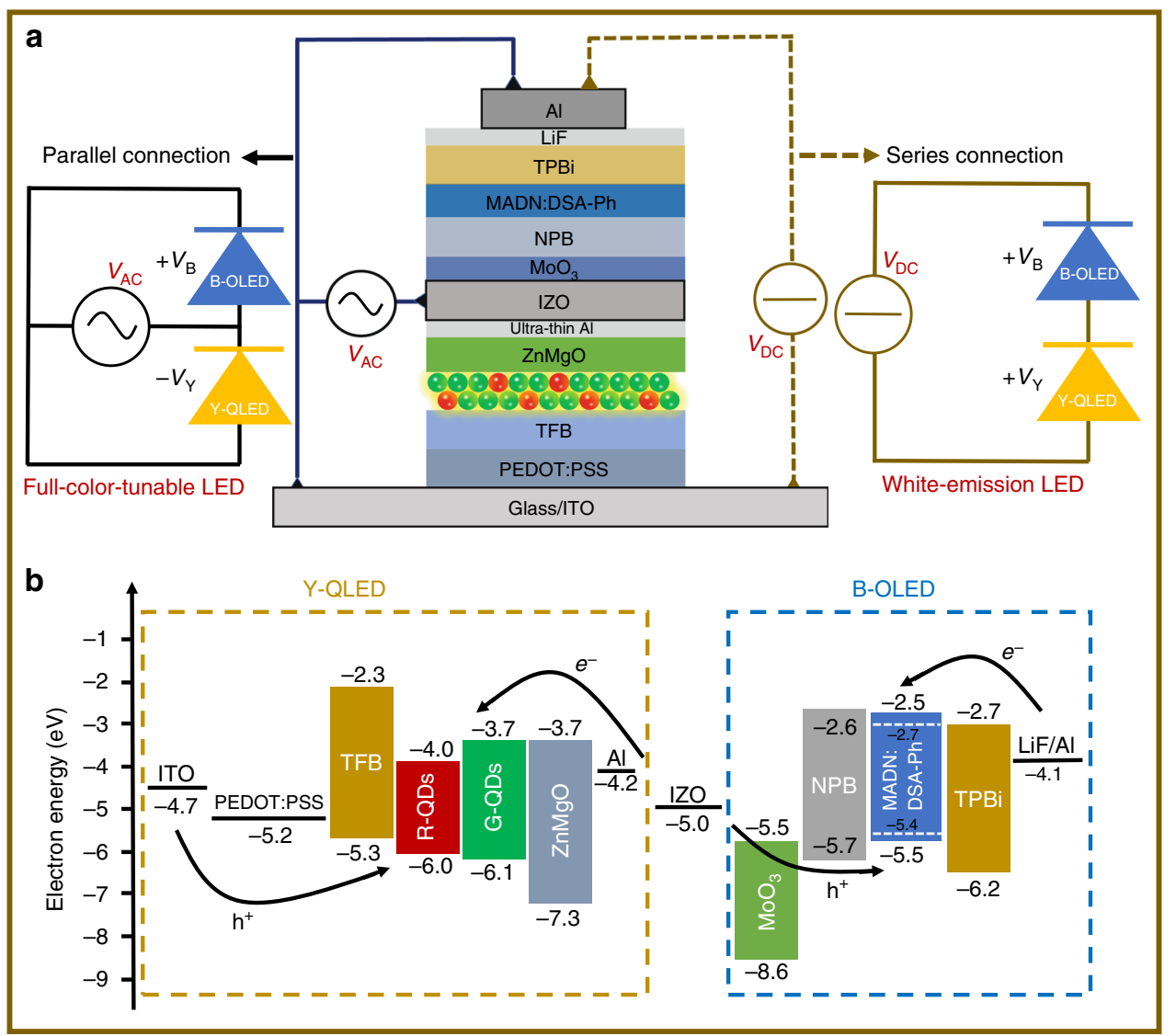

Fig. 1 Device structure and driving methods of the multifunctional LED. a Schematic device structure of the multifunctional tandem LED with an extractable IZO intermediate electrode. The Y-QLED and B-OLED can be connected either in parallel or in series. Under parallel connection and AC driving, a color-tunable device is achieved. Under series connection and DC driving, a white-emitting device is obtained. b Energy level alignment of the multifunctional tandem LED.

is controlled by the voltage, which can be tuned from pure $\mathrm{R}$ to pure $G$ by increasing the voltage. With such configuration, the emitting color of the tandem LED can be controlled by both polarity and amplitude of the driving signals. Therefore, by simply varying the driving $\mathrm{AC}$ signals, the tandem LED can alternately emit three primary colors, with the $\mathrm{B}$ color controlled by the positive pulses, the $\mathrm{R}$ and the $\mathrm{G}$ colors activated by the amplitude of the negative pulses. Also, arbitrary colors, located inside a color triangle defined by the primary colors, can be obtained by integrating the emission of both Y-QLED and B-OLED.

Figure $2 \mathrm{~b}-\mathrm{d}$ shows the driving signals, the CIE chart, the photos, and the emission spectra of a color-tunable tandem LED that emits different colors. To drive the device, an AC source with positive pulses $V_{\mathrm{B}}$ and negative pulses $V_{\mathrm{Y}}$, which control the luminance of $\mathrm{B}-\mathrm{OLED}$ and Y-QLED, respectively, was applied. The frequency of the AC source was set at $100 \mathrm{~Hz}$ so that human eyes perceive a combined emission from both B-OLED and Y-QLED. As shown in Fig. 2b, d, the color can be continuously tuned from:

(I) $\mathrm{R}$ to $\mathrm{G}$. In this case, only the Y-QLED is activated while the B-OLED is switched off by setting $V_{\mathrm{B}}=0$. By gradually increasing the $V_{\mathrm{Y}}$ from -2 to $-8 \mathrm{~V}$, the emission color is tuned from $\mathrm{R}, \mathrm{Y}$ to $\mathrm{G}$. The photos and the emission spectra of the tandem LED with different colors are displayed in Fig. $2 \mathrm{~b}$ and $\mathrm{d}$, respectively. Pure $\mathrm{R}$ emission with a color coordinate of $(0.67,0.32)$ is obtained at $2 \mathrm{~V}$, while the $\mathrm{G}$ emission is achieved at $8 \mathrm{~V}$ and its purity is affected by the blending ratio of R- and G-QDs. As shown in Supplementary Fig. 3, the purity of $G$ is degraded by the $R$ emission if excess R-QDs are incorporated. The purity of $\mathrm{G}$ is also affected by the thickness of IZO due to the microcavity effect, as shown in Supplementary Fig. 4. At an optimal RQDs:G-QDs mixing ratio of 1:20 and an IZO thickness of $60 \mathrm{~nm}$, the purest $\mathrm{G}$ emission with a color coordinate of $(0.26,0.29)$ is obtained.

(II) $\mathrm{B}$ to $\mathrm{R}$. The $\mathrm{B}$ emission is obtained by activating the $\mathrm{B}$ OLED only. When both B-OLED and Y-QLED are alternately turned-on, a new color resulting from the mixing of the emission of both devices is generated. To ensure a pure $\mathrm{R}$ emission, the $V_{\mathrm{Y}}$ is set at $-2 \mathrm{~V}$. By decreasing the $V_{\mathrm{B}}$ from 4 to $0 \mathrm{~V}$, the blue intensity is gradually reduced and thus the color is tuned from $\mathrm{B}(0.16$, $0.31)$, purple $(0.27,0.32)$ to $\mathrm{R}(0.67,0.32)$.

(III) $\mathrm{B}$ to orange $(\mathrm{O})$. The driving method is similar to that of (II). Except that the $V_{\mathrm{Y}}$ is set at $-2.8 \mathrm{~V}$ to produce an $\mathrm{O}$ emission. Also, to balance the light intensities of both devices, the maximum $V_{\mathrm{B}}$ is raised to $5 \mathrm{~V}$ and the duty ratio (DR) is increased to $80 \%$. The color is changed from $\mathrm{B}$ $(0.16,0.31)$, warm white $(0.37,0.40)$ to $\mathrm{O}(0.52,0.46)$ when the $V_{\mathrm{B}}$ is decreased from 5.5 to $0 \mathrm{~V}$.

(IV) $B$ to G. The driving method is similar to that of (III). Except that the $V_{\mathrm{Y}}$ is set at $-8 \mathrm{~V}$ to ensure a pure $\mathrm{G}$ emission, and the DR is further increased to $95 \%$. When the $V_{\mathrm{B}}$ is decreased from 10 to $0 \mathrm{~V}$, the colors are shifted from $\mathrm{B}$ $(0.16,0.31)$, cyan $(0.18,0.40)$ to $\mathrm{G}(0.26,0.29)$.

As shown in Fig. $2 \mathrm{~b}$, the colors that are achievable can cover a color triangle with a $63 \%$ NTSC (National Television System 

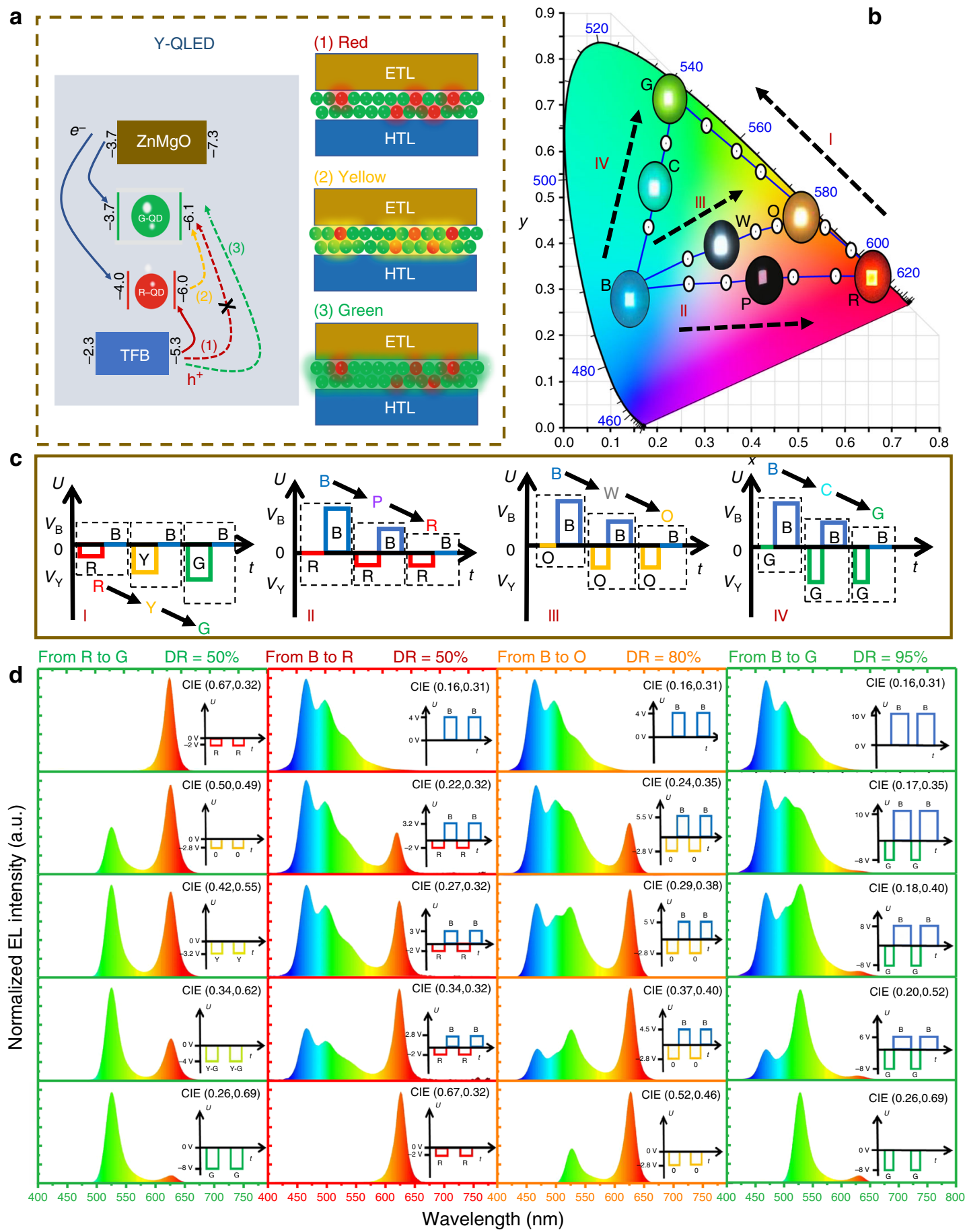

Fig. 2 Mechanism and driving methods of full-color-tunable LED. a Schematic diagram of the charge injection and recombination trajectory of the $Y$ QLED. The recombination zone is gradually migrated from R-QDs to G-QDs when the driving voltage is increased, and thus the emission color of Y-QLED can be controlled by the voltage. $\mathbf{b}$ The CIE chart, CIE coordinates, and photos; c the AC driving signals; and $\mathbf{d}$ the emission spectra of a color-tunable device that emits different colors. The color can be continuously tuned from (I) R to G, (II) B to R, (III) B to O, and (IV) B to G.

Committee) color gamut. To the best of our knowledge, this is the first demonstration of a full-color-tunable device that is simply driven by a single two-terminal source (also demonstrated in Supplementary Movie). The full-color-tunable device, which can take the job of three conventional R, G, and B sub-pixels, can enable a CF-free display with higher pixel density. To display a colorful picture, each frame can be divided into three sub-frames, corresponding to the R, G, and B colors of a picture. By fast and time-sequentially displaying the R, G, and B colors, a full-color picture can be synthesized. The gray level is also adjustable, since the B intensity can be independently controlled by the $V_{\mathrm{B}}$, while the $\mathrm{R}$ and $\mathrm{G}$ intensity can be altered by tuning the driving time.

The performance of B-OLED and Y-QLED can be separately evaluated by extracting the IZO electrode. As shown in Fig. 3a, a high EQE of $5.6 \%$ and $11.2 \%$ are achieved by B-OLED and Y-QLED, respectively, indicating that the introduction of IZO does not affect the performance adversely. The current density-voltage-luminance $(J-V-L)$ characteristics of the devices are shown in Fig. 3b. The B-OLED and Y-QLED exhibit a low turn-on voltage of 2.8 and $2.1 \mathrm{~V}$ and a maximum brightness of 

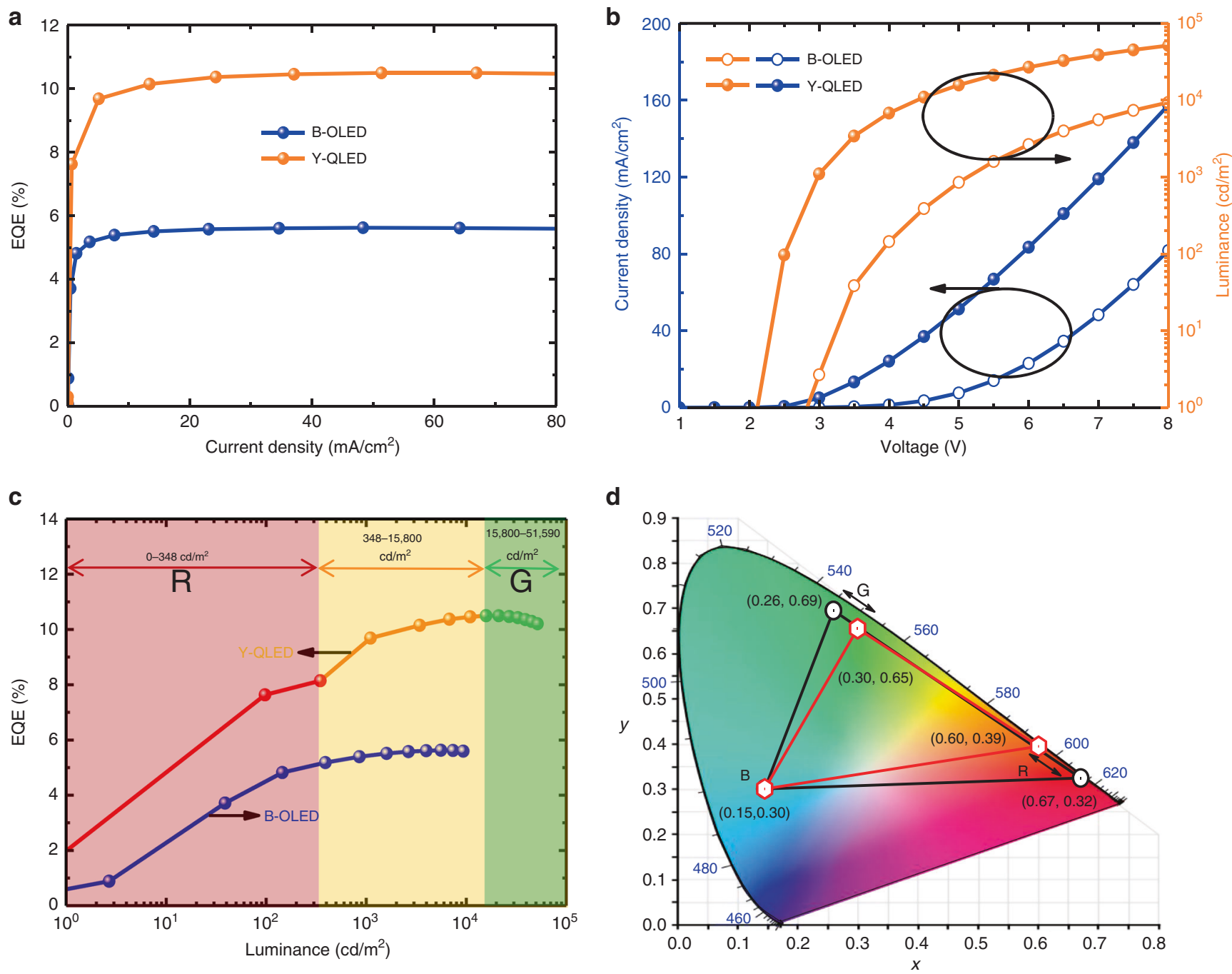

Fig. 3 Performance of the full-color-tunable LED. a The EQE-J, b J-V-L, and c EQE-L characteristics of B-OLED and Y-QLED, measured by extracting the IZO as a common electrode. d CIE chart and color coordinates of the R, G, and B primary colors. By degrading the color gamut from $63 \%$ to $45 \%$ NTSC, a wide range of brightness for the $R, G$, and $B$ emission can be obtained.

9359 and $51590 \mathrm{~cd} \mathrm{~m}^{-2}$, respectively. For the Y-QLED, the emission color is controlled by the driving voltage, and thus by correlating the emission color with the voltage, we can probe the brightness/efficiency of the R and G emission, as shown in Fig. 3c. The purest $\mathrm{R}$ emission with a CIE coordinate of $(0.67,0.32)$ is achieved at a low brightness of $0.35 \mathrm{~cd} \mathrm{~m}^{-2}$, which is too low for practical application. By losing the color saturation and moving the coordinate to $(0.60,0.39)$, the brightness and EQE can be enhanced to $348 \mathrm{~cd} \mathrm{~m}^{-2}$ and $8.2 \%$, respectively, which could be sufficient for display application. Similarly, for the $\mathrm{G}$ emission, if we allow the color saturation degrading from $(0.26,0.69)$ to $(0.30,0.65)$, the brightness can be decreased from 51,590 to $15,800 \mathrm{~cd} \mathrm{~m}^{-2}$, which could be further reduced to an application level by shorting the driving time in a frame period. In this case, the color gamut is decreased from $63 \%$ to $45 \%$ NTSC so that a wide range of brightness for the R, G, and B emission can be obtained, as shown in Fig. 3d. The color gamut can further be improved by enhancing the color saturation of the B-OLED. The detailed performances of the device are summarized in Table 1.

Efficient and color stable white LED. Under series connection and DC driving, both B-OLED and Y-QLED are turned-on simultaneously. By integrating the emission from both devices, an efficient white emission is achieved. As shown in Fig. $4 a$, b, the tandem LED exhibits a turn-on voltage of $4.16 \mathrm{~V}$, a brightness of $32575 \mathrm{~cd} \mathrm{~m}^{-2}$ at $100 \mathrm{~mA} \mathrm{~cm}^{-2}$, and a peak EQE of $16.91 \%$, which are nearly equal to the sum of those of the B-OLED $(3.1 \mathrm{~V}, 11260$ $\left.\mathrm{cd} \mathrm{m}^{-2}, 6.23 \%\right)$ and Y-QLED $\left(2.2 \mathrm{~V}, 21716 \mathrm{~cd} \mathrm{~m}^{-2}, 11.86 \%\right)$, indicating that both devices are effectively connected by the IZO. As shown in Supplementary Fig. 5, the EQE of the white LED can be further improved to $26.02 \%$ (power efficiency increased from 12.16 to $20.31 \mathrm{~lm} \mathrm{~W}^{-1}$ ) by replacing the FL B-OLED with mCPPO1:Firpic-based Ph B-OLED. The emission spectra of the white LED are shown in Fig. 4d, which are normalized to the peak of the $\mathrm{R}$ emission. Because the $\mathrm{B}$ and the $\mathrm{G}$ emission are turnedon at a relatively high voltage, their emission intensity are gradually enhanced when the driving voltage is increased. As a result, the color coordinates are shifted from $(0.42,0.33)$ to $(0.31,0.36)$ when the brightness is increased from 1000 to $107,000 \mathrm{~cd} \mathrm{~m}^{-2}$, as shown in Supplementary Fig. 6. Conventionally, it is very challenging to realize a color-stable white LED due to the migration of the exciton recombination zone as well as the difference of the driving voltage of $\mathrm{R}, \mathrm{G}$, and $\mathrm{B}$ emission ${ }^{41-44}$. Fortunately, with our novel tandem structure, we are able to improve the color stability by using an AC driving. Figure 4h shows the driving 
Table 1 The performances of the multifunctional tandem LED.

\begin{tabular}{|c|c|c|c|c|c|c|}
\hline \multirow{3}{*}{$\begin{array}{l}\text { Color } \\
\text { tunable }\end{array}$} & Parallel & Green & -5 to -8 & $15,800-51,590$ & 10.5 & $(0.30,0.65)-(0.26,0.69)$ \\
\hline & & Blue & $0-8$ & $0-9359$ & 5.6 & $(0.15,0.30)$ \\
\hline & & White with FL-OLED & $8-15$ & $2696-107,389$ & 16.9 & $(0.42,0.33)-(0.31,0.36)$ \\
\hline \multirow{2}{*}{$\begin{array}{l}\text { White } \\
\text { emission }\end{array}$} & Series & White with Ph-OLED & $7-15$ & $364-58,605$ & 26.02 & $(0.23,0.29)-(0.31,0.36)$ \\
\hline & Parallel & White with & $V_{Y}=-12$ & $1000-50,000$ & Y-QLED: 11.86 & $(0.34,0.36)$ \\
\hline
\end{tabular}
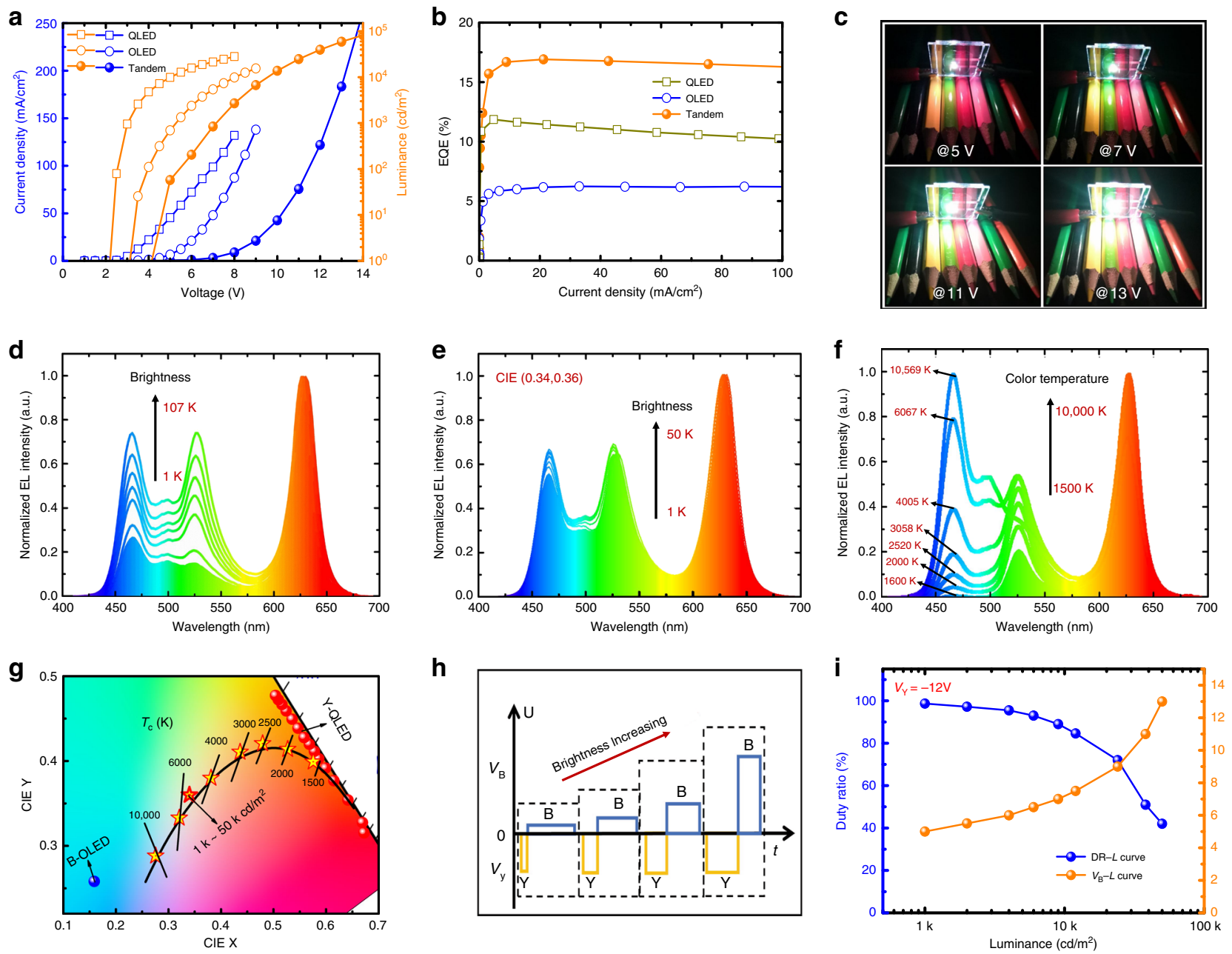

Fig. 4 Performance of the tandem white LED. a The $J-V-L$, b EQE- $J$ characteristics of the B-OLED, Y-QLED, and tandem white LED. c The photos of an operating tandem white LED driven by different voltage levels. d Normalized emission spectra of the tandem white LED under $\mathbf{d}$ DC driving, and e, $\mathbf{f}$ AC driving. $\mathbf{g}$ CIE coordinates of the white LED (star), B-OLED (blue point), and Y-QLED (red point). Under AC driving, stable white emission with CIE coordinates fixed at $(0.34,0.36)$ can be obtained for a wide range of brightness $\left(1000-50,000 \mathrm{~cd} \mathrm{~m}^{-2}\right)$. Also, the color temperature can be tuned from 1500 to $10,000 \mathrm{~K}$, which well traces the blackbody locus. $\mathbf{h} A C$ signals and $\mathbf{i}$ DR- $L$ and $V_{B}-L$ curves for driving a color stable white $L E D$.

signals for a color stable white LED. The $V_{\mathrm{Y}}$ for controlling the YQLED is fixed at $-12 \mathrm{~V}$ to ensure a stable $\mathrm{Y}$ emission. In this case, the brightness of the Y-QLED and B-OLED is controlled by the $\mathrm{DR}$ of the AC signals and the $V_{\mathrm{B}}$, respectively. By gradually increasing $V_{\mathrm{B}}$ and decreasing DR (Fig. 4i), a very stable white emission with a brightness increasing from 1000 to $50,000 \mathrm{~cd} \mathrm{~m}^{-2}$ is obtained. As shown in Fig. $4 \mathrm{~g}$, the color coordinates are fixed at $(0.34,0.36)$ for a wide range of brightness. The corresponding emission spectra are shown in Fig. 4e. Because the QDs have relatively narrow emission spectra and the emission of G-QDs peaked at $524 \mathrm{~nm}$ is optimized for display purpose, the resultant white emission exhibits a low color rendering index (CRI) of only 60 , which could be further improved by using G-QDs with redder emission (e.g. $550 \mathrm{~nm}$ ). Besides stable white emission for a wide range of brightness, the white LED can also be tuned to mimic the blackbody radiation. As shown in Fig. $4 \mathrm{f}$, by varying the $V_{\mathrm{Y}}$ and the $V_{\mathrm{B}}$, the emission intensity of Y-QLED and B-OLED can be tuned in a wide range, thereby allowing us to obtain a series of white emission with different ratios of yellow and blue emission intensity. Specifically, the color coordinates of the white LED can 
be tuned to trace the blackbody locus for a wide range of correlated color temperature $(1500-10,000 \mathrm{~K})$, as shown in Fig. $4 \mathrm{~g}$. The detailed performances of the white LED are shown in Table 1. The white LED, with high efficiency, high brightness, pure white color, high color stability, and tunable color temperature, could be the ideal lighting source for high-quality illumination, as demonstrated in Fig. 4c.

\section{Discussion}

In summary, a multifunctional hybrid tandem LED is demonstrated, which is realized by: (1) stacking a Y-QLED and a BOLED vertically, (2) using a conductive and transparent IZO as the ICE, and (3) configuring the EML of Y-QLED with mixing RQDs and G-QDs. With the novel IZO ICE, the Y-QLED and BOLED can be effectively connected either in parallel or in series, thus enabling the tandem LED to work multi-functionally. Under parallel connection, the tandem LED can be tuned to emit a wide range of colors, including R, G, and B primary colors, as well as arbitrary colors that are inside a color triangle defined by the primary colors. The achievable brightness of the R, G, and B primary colors could meet the requirement of the display application. With the color-tunable tandem LED, a full-color display with a color gamut up to $63 \%$ NTSC could be realized, and also, the absorptive CFs could be eliminated, the problematic EML patterning could be canceled and the pixel density could be improved by threefold. When under conventional series connection, the tandem LED exhibits an efficient white emission with a high brightness of $107,000 \mathrm{~cd} \mathrm{~m}^{-2}$ and an EQE up to $26.02 \%$. Under AC driving, stable white color $(0.34,0.36)$ over a wide range of brightness $\left(1000-50,000 \mathrm{~cd} \mathrm{~m}^{-2}\right)$, as well as tunable color temperature $(1500-10,000 \mathrm{~K})$ can be achieved. We believe that our demonstrated two-terminal tandem LED, with multifunctionality of full-color-tunability and white light emission, could find potential applications in both full-color display and solid-state lighting.

\section{Methods}

Device structures. Multifunctional tandem LEDs were fabricated by using the structure of glass/ITO/PEDOT:PSS $(45 \mathrm{~nm}) / \mathrm{TFB}(40 \mathrm{~nm}) / \mathrm{R}-\mathrm{QDs}$ :G-QDs $(18 \mathrm{~nm}$, 1:20 for color-tunable device or 1:9 for white device)/ZnMgO $(40 \mathrm{~nm}) / \mathrm{Al}(2 \mathrm{~nm}) /$ IZO $(60 \mathrm{~nm}$ or $80 \mathrm{~nm}) / \mathrm{MoO}_{3}(8 \mathrm{~nm}) / \mathrm{NPB}(45 \mathrm{~nm}) / \mathrm{MADN}: \mathrm{DSA}-\mathrm{Ph}(25 \mathrm{~nm}) / \mathrm{TPBi}$ $(40 \mathrm{~nm}) / \mathrm{LiF}(1 \mathrm{~nm}) / \mathrm{Al}(100 \mathrm{~nm})$ (abbreviations: PEDOT:PSS = poly(3,4-ethylenedioxythiophene $) /$ polystyrenesulfonate, TFB $=\operatorname{poly}[(9,9$-dioctylfluorenyl-2,7-diyl $)$ $\operatorname{co}\left(4,4^{\prime}\right.$ - $(N$-( $p$-butylphenyl))diphenylamine) $], \mathrm{NPB}=N, N^{\prime}$-bis-(1-naphthyl)- $N, N^{\prime}$ diphenyl-1,1'-biphenyl-4,4" -diamine, MADN = 2-methyl-9,10-di(2-naphthyl) anthracene, $\mathrm{DSA}-\mathrm{Ph}=p$-bis $(p-N, N$-diphenyl-aminostyryl $)$ benzene, $\mathrm{TPBi}=$ $2,2^{\prime}, 2^{\prime \prime}$-(1,3,5-benzinetriyl)-tris(1-phenyl-1-H-benzimidazole).

To improve the efficiency of white device, the phosphorescent B-OLED-based tandem LED with structure of glass/ITO/PEDOT:PSS $(45 \mathrm{~nm}) / \mathrm{TFB}(40 \mathrm{~nm}) / \mathrm{R}$ QDs:G-QDs $(18 \mathrm{~nm}, 1: 9) / \mathrm{ZnMgO}(40 \mathrm{~nm}) / \mathrm{Al}(2 \mathrm{~nm}) / \mathrm{IZO}(60 \mathrm{~nm}$ or $80 \mathrm{~nm}) /$ HATCN $(20 \mathrm{~nm}) /$ TAPC $(40 \mathrm{~nm}) / \mathrm{mCP}(5 \mathrm{~nm}) /$ Firpic:mCPPO1 $(30 \mathrm{~nm}) / \mathrm{TmPyPB}$ $(30 \mathrm{~nm}) / \mathrm{LiF}(1 \mathrm{~nm}) / \mathrm{Al}(100 \mathrm{~nm})$ was also fabricated. (abbreviations: HATCN $=$ dipyrazino[2,3-f: $2^{\prime}, 3^{\prime}$-h] quinoxaline-2,3,6,7,10,11-hexacarbonitrile, TAPC $=1,1$-bis [4-[N,N-di(p-tolyl) aminophenyl]cyclohexane, $\mathrm{mCP}=1,3$-bis(9-carbazolyl) benzene, Firpic $=\operatorname{Ir}(4,6-\mathrm{dFppy}) 2(\mathrm{pic}), \mathrm{mCPPO} 1=9-(3-(9 \mathrm{H}-$ carbazole- 9 - $\mathrm{yl})$ phenyl)-3-(dibromophenylphosphoryl)-9H-carbazole, TmPyPB = 1,3,5-Tri(mpyridin-3-ylphenyl)benzene).

Device fabrication. The cleaned ITO glass substrates with a sheet resistance of $25 \Omega \square^{-1}$ were treated with an $\mathrm{O}_{2}$ plasma for $10 \mathrm{~min}$. After $\mathrm{O}_{2}$ plasma treatment, the hole injection layer was deposited by spin-casting a PEDOT:PSS (Clevios AI 4083 ) at $3000 \mathrm{r}$.p.m. for $45 \mathrm{~s}$ and baked at $130{ }^{\circ} \mathrm{C}$ for $15 \mathrm{~min}$ on a hot plate. Then, the samples were transferred to a nitrogen-filled glovebox to sequentially fabricate the following layers. The hole transport layer was deposited by spin-casting a TFB solution ( $8 \mathrm{mg} \mathrm{mL}^{-1}$ in chlorobenzene) at 3000 r.p.m. for $40 \mathrm{~s}$ followed by annealing at $110^{\circ} \mathrm{C}$ for $15 \mathrm{~min}$. The QD EML was formed by spin-casting a mixed solution of R- and G-QDs (R-QDs: CdZnSe/ZnS/OT, core and shell $\sim 12.2 \mathrm{~nm}$; GQDs: CdZnSeS/ZnS/oleic acid, core and shell $\sim 11.6 \mathrm{~nm}$. The QDs were purchased from Suzhou Mesolight Inc.) at 3000 r.p.m. for 40 s, followed by baking at $100^{\circ} \mathrm{C}$ for $5 \mathrm{~min}$. The mixed QD solution was obtained by mixing a R-QD solution (10 $\mathrm{mg} \mathrm{mL}^{-1}$ in octane) with a G-QD solution $\left(10 \mathrm{mg} \mathrm{mL}^{-1}\right.$ in octane). The mixing ratio of R- and G-QDs can be tuned by varying the volume ratio of both solutions. After EML fabrication, the electron transport layer was deposited by spin-casting the $\mathrm{Zn}_{0.85} \mathrm{Mg}_{0.15} \mathrm{O}$ nanoparticles solution $\left(20 \mathrm{mg} \mathrm{mL}^{-1}\right.$ in ethanol) at 2500 r.p.m. for $40 \mathrm{~s}$ and backed at $100^{\circ} \mathrm{C}$ for $10 \mathrm{~min}$.

After that, the samples were transferred to a high-vacuum evaporation chamber to deposit a $2 \mathrm{~nm} \mathrm{Al}$ protective layer at a base pressure of $5 \times 10^{-4} \mathrm{~Pa}$. Then, the IZO with a thickness of $60 \mathrm{~nm}$ for color-tunable device or $80 \mathrm{~nm}$ for white tandem device was fabricated by a magnetron sputtering system at a working pressure of $0.45 \mathrm{~Pa}$, a power of $50 \mathrm{~W}$, an Ar flow of $20 \mathrm{sccm}$. The IZO target is composed of $90 \mathrm{wt} \% \mathrm{In}_{2} \mathrm{O}_{3}$ and $10 \mathrm{wt} \% \mathrm{ZnO}$. The sputtered 80 and $60 \mathrm{~nm}$ IZO films show the sheet resistance of $\sim 87 \Omega \square^{-1}$ and $\sim 116 \Omega \square^{-1}$, respectively. The IZO was patterned by using a shadow mask. Even with a 2-nm-thick Al, the Al/IZO electrode still exhibits a high transparency of $85 \%$ and a low reflectance of $8 \%$, as shown in Supplementary Fig. 7. After IZO fabrication, the samples were transferred to a high-vacuum evaporation chamber to deposit the OLED at a base pressure of $5 \times 10^{-4} \mathrm{~Pa}$. For fluorescent B-OLED, the layers of $\mathrm{MoO}_{3}(8 \mathrm{~nm}), \mathrm{NPB}(45 \mathrm{~nm})$, MADN:DSA-Ph $(12 \%, 25 \mathrm{~nm}), \mathrm{TPBi}(40 \mathrm{~nm}), \operatorname{LiF}(1 \mathrm{~nm})$, and $\mathrm{Al}(100 \mathrm{~nm})$ were sequentially deposited, and for phosphorescent B-OLED, the layers of HATCN $(20 \mathrm{~nm})$, TAPC $(40 \mathrm{~nm}), \mathrm{mCP}(5 \mathrm{~nm})$, Firpic:mCPPO1 (8\%, $30 \mathrm{~nm}), \mathrm{TmPyPB}$ $(30 \mathrm{~nm}), \operatorname{LiF}(1 \mathrm{~nm})$, and $\mathrm{Al}(100 \mathrm{~nm})$ were sequentially deposited. The layout of the tandem LED with bottom ITO, top Al, and intermediate IZO electrodes are shown in Supplementary Fig. 8.

Device characterization. The thicknesses of IZO films and all the solutionprocessed films were measured by using a Bruker DektakXT Stylus Profiler. The AC square-wave voltage signals were provided by a waveform generator (JunCe JDS-6600). The evaporation rates and the thicknesses of all the organic layers, $\mathrm{MoO}_{3}, \mathrm{LiF}$, and $\mathrm{Al}$ were in situ monitored by a quartz crystal microbalance. The emission spectra of all the devices were measured by a PR670 spectrometer. The $J-V-L$ characteristics were characterized by a programmable source meter (Keithley 2614B) and a PR670 spectrometer. Because of the high transparency of the Al/IZO ICE (Supplementary Fig. 7), the microcavity effect is quite weak. Considering the weak microcavity effect, the $\mathrm{EQE}$ was calculated by assuming that the emission is Lambertian. The calculated EQE was further verified by a method recommended by SR Forrest ${ }^{45}$. A large-area $\left(613 \mathrm{~mm}^{2}\right)$ PIN-25D silicon photodiode was placed in close contact with the devices (active area $2 \mathrm{~mm} \times 2 \mathrm{~mm}$ ). By measuring the photodiode current and the driving current, the EQE (as photons per electron) was calculated by converting the photodiode current to emitted photons and the driving current to electrons.

\section{Data availability}

The data that support the findings of this study are available from the corresponding author upon reasonable request.

Received: 13 March 2020; Accepted: 12 May 2020;

Published online: 04 June 2020

\section{References}

1. Dai, X. et al. Solution-processed, high-performance light-emitting diodes based on quantum dots. Nature 515, 96-99 (2014).

2. Yang, Y. et al. High-efficiency light-emitting devices based on quantum dots with tailored nanostructures. Nat. Photon 9, 259-266 (2015).

3. Shen, H. et al. Visible quantum dot light-emitting diodes with simultaneous high brightness and efficiency. Nat. Photon 13, 192-197 (2019).

4. Colvin, V. L., Schlamp, M. C. \& Alivisatos, A. P. Light-emitting-diodes made from cadmium selenide nanocrystals and a semiconducting polymer. Nature 370, 354-357 (1994).

5. Coe, S., Woo, W.-K., Bawendi, M. \& Bulović, V. Electroluminescence from single monolayers of nanocrystals in molecular organic devices. Nature 420, 800-803 (2002).

6. Sun, Y. et al. Investigation on thermally induced efficiency roll-off: toward efficient and ultrabright quantum-dot light-emitting diodes. ACS Nano 13, 11433-11442 (2019).

7. Mashford, B. S. et al. High-efficiency quantum-dot light-emitting devices with enhanced charge injection. Nat. Photon 7, 407-412 (2013).

8. $\mathrm{Li}, \mathrm{X}$. et al. Bright colloidal quantum dot light-emitting diodes enabled by efficient chlorination. Nat. Photon 12, 159-164 (2018).

9. Dai, X., Deng, Y., Peng, X. \& Jin, Y. Quantum-dot light-emitting diodes for large-area displays: towards the dawn of commercialization. Adv. Mater. 29, 1607022 (2017)

10. Qian, L., Zheng, Y., Xue, J. \& Holloway, P. H. Stable and efficient quantumdot light-emitting diodes based on solution-processed multilayer structures. Nat. Photon 5, 543-548 (2011).

11. Shirasaki, Y., Supran, G. J., Bawendi, M. G. \& Bulović, V. Emergence of colloidal quantum-dot light-emitting technologies. Nat. Photon 7, 13-23 (2013). 
12. Cho, K.-S. et al. High-performance crosslinked colloidal quantum-dot lightemitting diodes. Nat. Photon 3, 341-345 (2009).

13. Song, J. et al. Over $30 \%$ external quantum efficiency light-emitting diodes by engineering quantum dot-assisted energy level match for hole transport layer. Adv. Funct. Mater. 29, 1808377 (2019).

14. Zhang, H., Chen, S. \& Sun, X. W. Efficient red/green/blue tandem quantumdot light-emitting diodes with external quantum efficiency exceeding $21 \%$. ACS Nano 12, 697-704 (2018).

15. Yang, Z. et al. All-solution processed inverted green quantum dot lightemitting diodes with concurrent high efficiency and long lifetime. Mater. Horiz. 6, 2009-2015 (2019).

16. Moon, H. \& Chae, H. Efficiency enhancement of all-solution-processed inverted-structure green quantum dot light-emitting diodes via partial ligand exchange with thiophenol derivatives having negative dipole moment. Adv. Opt. Mater. 8, 1901314 (2020).

17. Zhang, H., Sun, X. W. \& Chen, S. Over $100 \mathrm{~cd} \mathrm{~A}^{-1}$ efficient quantum dot light-emitting diodes with inverted tandem structure. Adv. Funct. Mater. 27, 1700610 (2017).

18. Li, X. et al. Quantum-dot light-emitting diodes for outdoor displays with high stability at high brightness. Adv. Opt. Mater. 8, 1901145 (2020).

19. Wang, O. et al. High-efficiency, deep blue $\mathrm{ZnCdS} / \mathrm{Cd}_{\mathrm{x}} \mathrm{Zn}_{1-\mathrm{x}} \mathrm{S} / \mathrm{ZnS}$ quantumdot-light-emitting devices with an EQE exceeding 18\%. Nanoscale 10, 5650-5657 (2018).

20. Cao, W. et al. Highly stable QLEDs with improved hole injection via quantum dot structure tailoring. Nat. Commun. 9, 1-6 (2018).

21. $\mathrm{Pu}, \mathrm{C}$. et al. Electrochemically-stable ligands bridge the photoluminescenceelectroluminescence gap of quantum dots. Nat. Commun. 11, 1-10 (2020).

22. Liu, Y. et al. Efficient all-solution processed quantum dot light emitting diodes based on inkjet printing technique. ACS Appl. Mater. Interfaces 9, 25506-25512 (2017).

23. Kim, T.-H. et al. Full-colour quantum dot displays fabricated by transfer printing. Nat. Photon 5, 176-182 (2011).

24. Yang, P., Zhang, L., Kang, D. J., Strahl, R. \& Kraus, T. High-resolution inkjet printing of quantum dot light-emitting microdiode arrays. Adv. Opt. Mater. 8, 1901429 (2020).

25. Wood, V. et al. Inkjet-printed quantum dot-polymer composites for full-color AC-driven displays. Adv. Mater. 21, 1-5 (2009).

26. Kwon, J. H. RGB color patterning for AMOLED TVs. Inf. Disp. 29, 12-15 (2013).

27. Han, C.-W., Tak, Y.-H. \& Ahn, B.-C. 15-in. RGBW panel using two-stacked white OLED and color filters for large-sized display applications. J. Soc. Inf. Disp. 19, 190-195 (2011)

28. Asaki, R. et al. A 0.23-in. High-resolution OLED microdisplay for wearable displays. SID Int. Symp. Dig. Tech. Pap. 45, 219-222 (2014).

29. Prache, O. Full-color SVGA+ OLED-on-silicon microdisplay. J. Soc. Inf. Disp. 10, 133-138 (2002).

30. Shen, Z., Burrows, P. E., Bulović, V., Forrest, S. R. \& Thompson, M. E. Threecolor, tunable, organic light-emitting devices. Science 276, 2009-2011 (1997).

31. Parthasarathy, G., Gu, G. \& Forrest, S. R. A full-color transparent metal-free stacked organic light emitting device with simplified pixel biasing. Adv. Mater. 11, 907-910 (1999).

32. Cheng, G., Chan, K. T., To, W.-P. \& Che, C.-M. Color tunable organic lightemitting devices with external quantum efficiency over $20 \%$ based on strongly luminescent gold(III) complexes having long-lived emissive excited states. Adv. Mater. 26, 2540-2546 (2014).

33. Guo, F. et al. The fabrication of color-tunable organic light-emitting diode displays via solution processing. Light.: Sci. Appl 6, e17094 (2017).

34. Fröbel, M. et al. Get it white: color-tunable AC/DC OLEDs. Light Sci. Appl. 4, e247 (2015).

35. Zhang, J. et al. Voltage-dependent multicolor electroluminescent device based on halide perovskite and chalcogenide quantum-dots emitters. Adv. Funct. Mater. 30, 1907074 (2020).

36. Qasim, K. et al. A color tunable quantum-dot light-emitting diode device driven by variable voltage. J. Nanosci. Nanotechnol. 19, 1038-1043 (2019).

37. Jiang, C. et al. Fully solution-processed tandem white quantum-dot lightemitting diode with an external quantum efficiency exceeding $25 \%$. ACS Nano 12, 6040-6049 (2018).
38. Fung, M.-K., Li, Y.-Q. \& Liao, L.-S. Tandem organic light-emitting diodes. Adv. Mater. 28, 10381-10408 (2016).

39. Choi, M. K. et al. Extremely vivid, highly transparent, and ultrathin quantum dot light-emitting diodes. Adv. Mater. 30, 1703279 (2018).

40. Zhang, H. \& Chen, S. ZnMgO:PVP inorganic-organic hybrid electron transport layer: towards efficient bottom-emission and transparent quantum dot light-emitting diodes. J. Mater. Chem. C. 7, 2291-2298 (2019).

41. Zhang, H., Su, Q., Sun, Y. \& Chen, S. Efficient and color stable white quantum-dot light-emitting diodes with external quantum efficiency over 23\%. Adv. Opt. Mater. 6, 1800354 (2018).

42. Lee, K.-H. et al. Highly efficient, color-reproducible full-color electroluminescent devices based on red/green/blue quantum dot-mixed multilayer. ACS Nano 9, 10941-10949 (2015).

43. Bae, W. K. et al. R/G/B/Natural white light thin colloidal quantum dot-based light-emitting devices. Adv. Mater. 26, 6387-6393 (2014).

44. Lee, K.-H. et al. Full-color-capable light-emitting diode based on solution processed quantum dot layer stacking. Nanoscale 10, 6300-6305 (2018).

45. Forrest, S. R., Bradley, D. D. \& Thompson, M. E. Measuring the efficiency of organic light emitting devices. Adv. Mater. 15, 1043-1048 (2003).

\section{Acknowledgements}

This work was supported by the National Natural Science Foundation of China (61775090), the Guangdong Natural Science Funds for Distinguished Young Scholars (2016A030306017), and the Guangdong Special Funds for Science and Technology Development (2017A050506001)

\section{Author contributions}

S.C. conceived the idea, supervised the work, and wrote the paper. H.Z. conducted the experiments, collected the data, and drew the figures. S.C., H.Z., and Q.S. discussed the results and commented on the manuscript.

\section{Competing interests}

The authors declare no competing interests.

\section{Additional information}

Supplementary information is available for this paper at https://doi.org/10.1038/s41467020-16659-x.

Correspondence and requests for materials should be addressed to S.C.

Peer review information: Nature Communications thanks Bjorn Lussem, Heesun Yang and the other, anonymous, reviewer(s) for their contribution to the peer review of this work. Peer reviewer reports are available.

Reprints and permission information is available at http://www.nature.com/reprints

Publisher's note Springer Nature remains neutral with regard to jurisdictional claims in published maps and institutional affiliations.

Open Access This article is licensed under a Creative Commons Attribution 4.0 International License, which permits use, sharing, adaptation, distribution and reproduction in any medium or format, as long as you give appropriate credit to the original author(s) and the source, provide a link to the Creative Commons license, and indicate if changes were made. The images or other third party material in this article are included in the article's Creative Commons license, unless indicated otherwise in a credit line to the material. If material is not included in the article's Creative Commons license and your intended use is not permitted by statutory regulation or exceeds the permitted use, you will need to obtain permission directly from the copyright holder. To view a copy of this license, visit http://creativecommons.org/ licenses/by/4.0/

(C) The Author(s) 2020 